\title{
Field and flow-based separations
}

\author{
Gaëtane Lespes $^{1} \cdot$ Catia Contado $^{2} \cdot$ Bruce K. Gale $^{3}$
}

Published online: 30 April 2015

(C) Springer-Verlag Berlin Heidelberg 2015

Field-flow fractionation (FFF) and related methods are a set of analytical methods for the separation and characterization of nano- and micro-size analytes. These methods were originally used for the characterization of synthetic polymers and biological macromolecules, before also providing important contributions to the field of environmental sciences. In the last 15 years FFF has benefitted from renewed interest, especially for the characterization of new analytes, from the nanosciences, where the production of objects of different shapes and structures and of increasingly small sizes is an ongoing objective. The synthesis or the development of new particles involves diverse applications, ranging from the environment through medicine to food and industrial applications. This diversity ensures the interest of very different and heterogeneous scientific communities in FFF, and these communities were all brought together in the recent FFF International Symposia.

The main recent FFF investigations are linked to nanotechnology (e.g. nanoparticles, polymers of very high molecular weight) and medicine (e.g. proteins, lipids, exosomes, liposomes, cells), with a strong interaction between the two in

Published in the topical collection Field- and Flow-based Separations with guest editors Gaetane Lespes, Catia Contado, and Bruce Gale.

Gaëtane Lespes

gaetane.lespes@univ-pau.fr

1 IPREM UMR5254, Technopôle Hélioparc Pau Pyrénées, 2, avenue du Président Angot Pau, 64053 Pau Cedex 09, France

2 Dipartimento di Scienze Chimiche e Farmaceutiche, Università degli Studi di Ferrara, Via Luigi Borsari 46, 44121 Ferrara, Italy

3 Department of Mechanical Engineering, University of Utah, $50 \mathrm{~S}$. Central Campus Dr Room 2110, Salt Lake City, UT 84112, USA nano-medicine. The needs and perspectives of these fields are stimulating important new research efforts. Indeed, the ability to describe nano- and micro-objects, from the elementary level to micrometer scale, enables a better understanding of the processes from which they come and in which they are involved. For these efforts, methods based on the coupling between field and flow have been found to be particularly effective in terms of selectivity and operational flexibility. Many FFF-related techniques exist, based on different types of applied field, including hydrodynamics, sedimentation, thermal, electrical, magnetic, acoustic, and more. These techniques have resulted in numerous works directly connected with the socio-technological concerns of the moment. Several papers were presented at recent FFF symposiums using all of these techniques and addressing many of these concerns. Among these techniques, asymmetric-flow fieldflow fractionation (AF4) remains the most used. Because of its non-selective force field, AF4 is applicable to all types of analyte, making it the most versatile of FFF techniques. Additionally, this technique seems to be easily hyphenated to detectors of very different natures. It is particularly well suited to coupling with light-scattering detectors (LS). Thus AF4-LS is revealed to be a powerful tool for dimensional analysis of nano-objects of any nature: molecular, supramolecular, or particulate. AF4 may also be coupled with online detectors including UV or molecular fluorescence, enabling the characterization of organic or inorganic objects of different origins, which has proved to be an advantage, especially for studying environmental and biological media. A variant of flow-FFF proposed a few years ago, the hollow fiber, has been found to have substantial potential in terms of selectivity and, especially, reduction of the sample volume. Whatever the technique, analytical investigation accompanies theoretical studies whose objective is a better understanding of the behavior of the separation system and retention processes. 
The articles related to FFF techniques contained in this topical collection and presented in the last two symposia organized, the first in Pau (France) in 2013 and the second in Salt Lake City (Utah, USA) in 2014, reflect current concerns regarding characterization and recent advances.

As guest editors of this issue, we are all very grateful to all contributing authors for their willingness to submit their manuscripts to this journal and we hope that reading these articles will provide information that can be reapplied to your own characterization challenges. We also hope that this collection of articles contributes to the development of FFF for the characterization of new nanoscale objects, reinforcing FFF's place in nanometrology and in validation processes and in stimulating new instrument development and new instrument combinations, new methods, and new fields of application. We would also thank the reviewers and the publishing team at Analytical and Bioanalytical Chemistry for their cooperation.

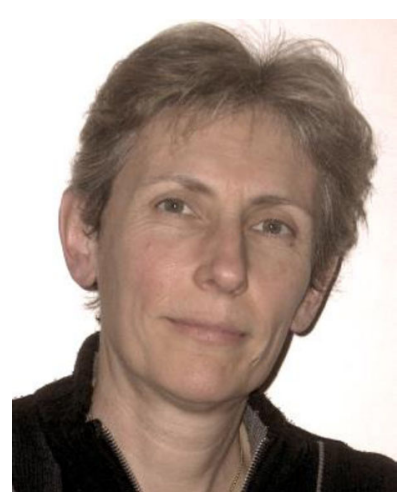

Gaëtane Lespes is a Professor in the Analytical Institute for Environmental and Material Sciences (IPREM) at the University of Pau et les Pays de l'Adour (UPPA). Her research focus is on the development of multitechnique characterization strategies based on field-flow fractionation and coupling with light scattering and atomic mass spectrometry. Her main research question is the physicochemical description of the fate of nanoparticles, polymers, and associated trace elements in complex media of environmental and biological interest. She created and is at the head of the group FFF (G4-F), within the International French-Speaking Association of Separation Sciences (AFSEP).

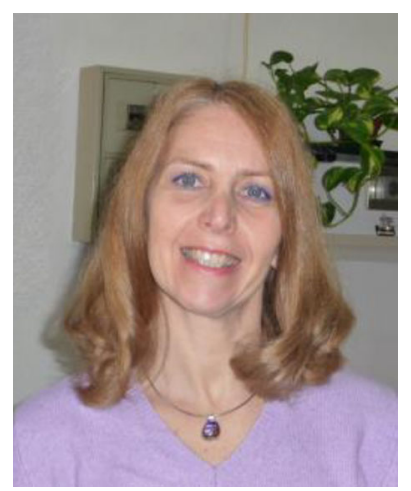

Catia Contado is a Researcher in Analytical Chemistry at the University of Ferrara. Her attention to FFF techniques began as a result of her interest in natural colloids and water-borne river particles analyses, whose characterization requires a combination of different analytical techniques including atomic absorption spectroscopy and inductively-coupledplasma atomic emission spectroscopy. Over the years, her research has moved to the characterization of micro and nanoparticles contained in consumer products and in pharmaceutical formulations, for which her previously acquired expertise has proved to be very useful.

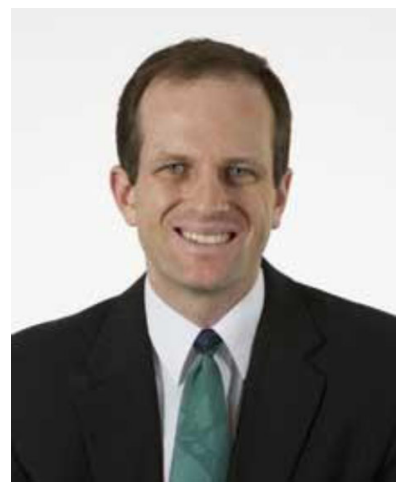

Bruce K. Gale is a Professor of Mechanical Engineering at the University of Utah and is currently Director of the Utah State Center of Excellence for Biomedical Microfluidics and the College of Engineering Nanofabrication Facility. He has been working in the field of FFF, microfluidics, nanotechnology, medical devices, and micro-total-analysis systems ( $\mu$-TAS) for the past 18 years. His primary interests include labon-a chip devices that require a variety of microfluidic components for the completion of complex and challenging medical and biological assays. Specifically, he is working to develop a microfluidic toolbox for the rapid design, simulation, and fabrication of devices with medical and biological applications. The ultimate objective is to develop techniques for personalized medicine, which should enable medical treatments to be customized to the needs of individual patients. 